\title{
Correspondence
}

Journal of Medical Genetics, 1981 ,18, 158-159

\section{Birth frequency of bilateral renal agenesis}

SIR,

In a family study of renal agenesis ( $\mathrm{J}$ Med Genet 1979; 16: 176-88), we reported an estimate of the birth frequency in 1974 of bilateral renal agenesis, based on death and stillbirth certificates supplied by OPCS, which gave Potter's syndrome, or a not fully specified renal anomaly or agenesis, as an underlying or one of multiple causes of death. For the deaths from 'multiple causes', we had only a $25 \%$ sample of certificates and we had no information on stillbirths because of 'multiple causes'; but for deaths and stillbirths where the underlying cause was renal agenesis, we had a $100 \%$ sample. The diagnosis was confirmed from necropsy reports in 50 cases. In four instances where a necropsy had been performed, but no report was available, we included the case because the death certificate or the paediatrician stated the cause of death to be renal agenesis. In two instances where no necropsy had been performed, but the death certificate stated Potter's syndrome and congenital abnormalities, we scored each case as a $\frac{1}{2}$ only. The estimated birth frequency was $0 \cdot 122$ per 1000 total births.

We now report data, applying the same criteria, for the three subsequent years. These are shown in the table together with the data for 1974. In 1977, as in 1974 , only a $25 \%$ sample of deaths from multiple causes was available and so the number was multiplied by 4 .

The birth frequency per thousand total births for each of the four years is: $1974,0 \cdot 122 ; 1975,0.096$; $1976,0.095 ; 1977,0 \cdot 120$. The figures for 1974 and 1975 do not include stillbirths from multiple causes. If, say, an allowance of three further cases is made for this deficit, the birth frequency estimate for $1974 \mathrm{D}$ and 1975 would be $0 \cdot 127$ and $0 \cdot 101$, respectively. Inevitably these figures will be somewhat of $a^{\text {s }}$ underestimate.

\section{O CARTER AND Kathleen Evans MRC Clinical Genetics Unit, Institute of Child Health, 30 Guilford Street, London WCIN IEHo}

\section{Familial partial 14 trisomy}

Sir,

A familial partial 14 trisomy was reported in the February 1979 issue of the Journal. ${ }^{1}$ On the basis $=$ of banding there were four affected subjects, $47,+\vec{\varphi}$ $\operatorname{der}(14), \mathrm{t}(9 ; 14)(\mathrm{p} 24 ; \mathrm{q} 21)$, and three maternal trans- location carriers $\mathrm{t}(9 ; 14)(\mathrm{p} 24 ; \mathrm{q} 21)$. The chromosomalo rearrangement includes triplication of the locus foro the enzyme nucleoside phosphorylase (NP). Georges and Francke ${ }^{2}$ have found that NP (purine-nucleoside:orthophosphate ribosyltransferase, EC 2.4.2.1)용 activity expressed in red blood cells is proportiona $\mathrm{Q}$ to the number of alleles present. They assayed $\overrightarrow{\overrightarrow{0}}$ erythrocytes from a series of normal controls and 3 subjects with partial 14 duplications to localise this enzyme. Three patients with partial 14 trisomy had NP activities of $21 \cdot 9,21 \cdot 8$, and 18.9 units of activity/g haemoglobin and were thought to demonso strate the activity of a triple gene dose. Sixty-ones normal persons had a mean activity of $12 \cdot 7$ units. range $7 \cdot 9$ to $18 \cdot 0$. With this assay procedure, our? patient DT had 21.7 units of NP activity/g haemoglobin (mean of eight normal people $15 \cdot 5$, range $13 \cdot 0$ to $17 \cdot 9$ ). These results support the reported karyotypes, the mapping of NP to region $14 q 13, \stackrel{3}{=}$.

TABLE Birth frequency of bilateral renal agenesis estimated from death and stillbirth certificates (England and Wales)

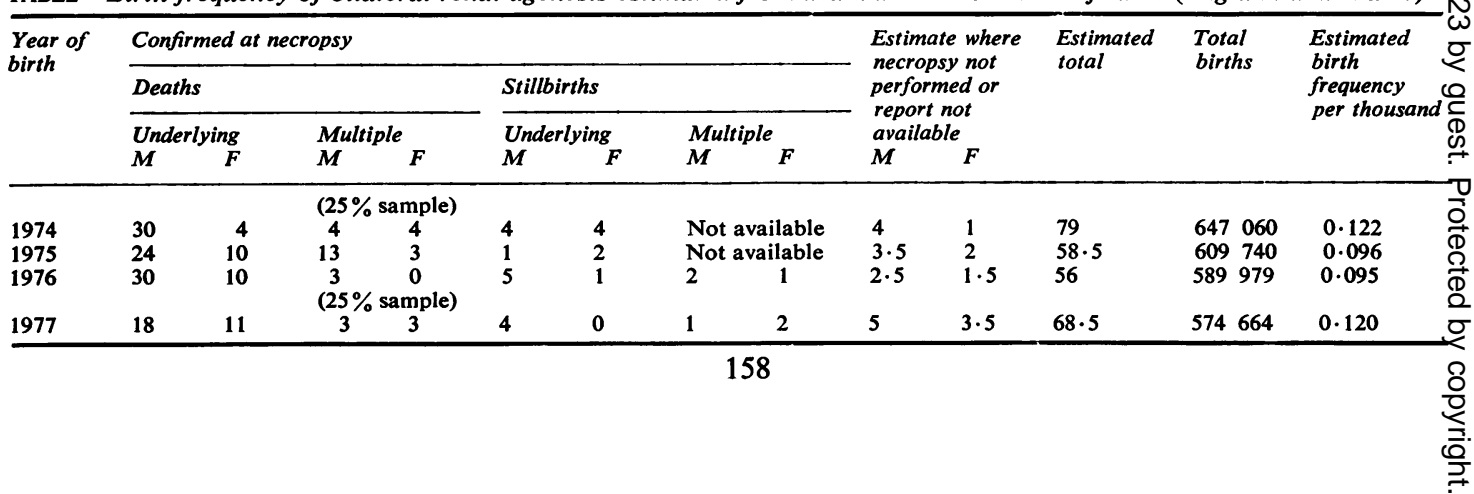

